\title{
Minocycline for the Treatment of Multidrug and Extensively Drug-Resistant $A$. baumannii: A Review
}

Jennifer N. Lashinsky • Oryan Henig · Jason M. Pogue •

Keith S. Kaye

Received: February 6, 2017 / Published online: March 29, 2017

(c) The Author(s) 2017. This article is an open access publication

\section{ABSTRACT}

Acinetobacter baumannii can cause life-threatening nosocomial infections associated with high rates of morbidity and mortality. In recent years, the increasing number of infections due to extensively drug-resistant Acinetobacter with limited treatment options has resulted in a need for additional therapeutic agents, and a renaissance of older, neglected antimicrobials. This has led to an increased interest in the use of minocycline to treat these infections. Minocycline has been shown to overcome many resistance mechanisms affecting other tetracyclines in $A$. baumannii, including tigecycline. Additionally, it has favorable pharmacokinetic and

Enhanced content To view enhanced content for this article go to http://www.medengine.com/Redeem/ 29F7F0607D680466.

J. N. Lashinsky $(\varangle)$

Department of Pharmacy, Barnes-Jewish Hospital, Saint Louis, MO, USA

e-mail: jennifer.lashinsky@bjc.org

O. Henig · K. S. Kaye

Department of Medicine, University of Michigan

Medical School, Ann Arbor, MI, USA

J. M. Pogue

Department of Pharmacy Services, Sinai-Grace

Hospital, Detroit Medical Center, Detroit, MI, USA

J. M. Pogue

Wayne State University School of Medicine, Detroit, MI, USA pharmacodynamic properties, as well as excellent in vitro activity against drug-resistant $A$. baumannii. Available data support therapeutic success with minocycline, while ease of dosing with no need for renal or hepatic dose adjustments and improved safety have made it an appealing therapy. This review will focus on the mechanisms of action and resistance to tetracyclines in A. baumannii, the in vitro activity, pharmacokinetic and pharmacodynamic properties of minocycline against $A$. baumannii, and finally the clinical experience with minocycline for the treatment of invasive infections due to this pathogen.

Keywords: Acinetobacter; Acinetobacter baumannii; Carbapenem resistance; Extensively drug-resistant; Minocycline; Multidrugresistant; Tetracyclines

\section{INTRODUCTION}

Acinetobacter baumannii and other Acinetobacter species can cause multidrug-resistant (MDR) nosocomial infections, with high morbidity and mortality rates. MDR A. baumannii, defined as $A$. baumannii resistant to more than 3 classes of antimicrobial agents, has been responsible for a variety of healthcare-associated infections within the last two decades $[1,2]$. Its ability to resist environmental stress and cleaning 
methods, and to acquire resistance to multiple classes of antimicrobial agents, have made this pathogen more common, and has become one of the most difficult to manage causes of outbreaks in the setting of intensive care units (ICUs) [3, 4]. In the Centers for Disease Control and Prevention (CDC) Antibiotic Resistance Threats report of 2013, MDR A. baumannii was declared a "serious threat" to public health in the United States [5]. Even more concerning has been the emergence of extensively drug-resistant or XDR strains of $A$. baumannii that are resistant to all but one or two classes of antimicrobials, and truly pan-drug-resistant strains resistant to all tested antimicrobials.

Traditionally, carbapenems were considered the drugs of choice when treating these highly resistant $A$. baumannii, but in recent years the widespread use of these agents has diminished their clinical activity. Carbapenems were considered appropriate agents to treat infections caused by MDR $A$. baumannii strains, but carbapenem-resistant $A$. baumannii (CRAB) has rapidly increased in frequency. CRAB accounts for $65 \%$ of $A$. baumannii pneumonia in the United States and Europe, while more than $60 \%$ of isolates in Asia have been found to be both pan-drug and carbapenem-resistant [6]. An analysis of $A$. baumannii performed in Detroit, MI reported decreasing susceptibility to therapies such as ampicillin-sulbactam and carbapenems in a rapid fashion. In this study, susceptibility of $A$. baumannii to ampicillin-sulbactam decreased from $89 \%$ to $40 \%$, while susceptibility to imipenem decreased from $99 \%$ to $42 \%$ from 2003 to 2008 [7]. The high resistance rates of these organisms has led to a situation with limited therapeutic options, where in vitro activity is frequently limited to the polymyxins, aminoglycosides, tigecycline and minocycline.

In the setting of carbapenem resistance, sulbactam (typically administered as ampicillin-sulbactam) is potentially an option for the treatment of $A$. baumannii; however, optimal dosing strategies for sulbactam remain poorly defined and resistance is increasing. Polymyxins are often considered an important treatment option for CRAB in the setting of sulbactam resistance, but unacceptably high nephrotoxicity rates and an inability to safely achieve pharmacodynamic targets has raised concern regarding the use of these agents. In mouse lung models of $A$. baumannii pneumonia, colistin was unable to achieve bacteriostasis even with the maximum tolerated doses against 2 of the 3 strains tested [8]. Furthermore, data suggest a clinically relevant increase in resistance of A. baumannii to colistin [9-11]. A recent surveillance study in the United States showed that $5.3 \%$ of all Acinetobacter strains were resistant to colistin [9], while another surveillance study in Greece reported an increase in colistin resistance from $1 \%$ in 2012 to $21.1 \%$ in 2014 among 1116 CRAB isolates [10]. An analysis from the Eurofins Surveillance Network demonstrated that resistance to carbapenems among Acinetobacter more than doubled in recent years $(21.0 \%$ in $2003-2005$ and $47.9 \%$ in 2009-2012) as did resistance to colistin (2.8\% in 2006-2008 and 6.9\% in 2009-2012). This same analysis demonstrated that rates of resistance to minocycline actually decreased over this time period from $56.5 \%$ in $2003-2005$ to $30.5 \%$ in 2009-2012 [11]. Currently, when CRAB infections demonstrate resistance to ampicillin-sulbactam (up to $74 \%$ in the SENTRY program were not susceptible to ampicillin-sulbactam per CLSI breakpoints) [12] and to colistin, the remaining alternatives for treatment are extremely limited.

While certain aminoglycosides can retain activity in vitro against CRAB, data suggest that aminoglycoside monotherapy is inadequate for infections occurring outside the urinary tract, and concerns remain regarding nephrotoxicity associated with this class. A meta-analysis reported that patients receiving aminoglycoside therapy had higher mortality rates and higher rates of microbiological failure than patients receiving beta-lactams or fluoroquinolones [13]. Antimicrobials such as tigecycline, ceftolazone-tazobactam and ceftazidime-avibactam have more recently come to market; but, unfortunately, these newer agents are not ideal for CRAB. Ceftolazone-tazobactam and ceftazidime-avibactam have minimal utility in the treatment of CRAB, given the usual mechanisms of resistance to carbapenems are class D or B carbapenemases. Both of these 
carbapenemases readily hydrolyze these cephalosporins and these enzymes are not inhibited by either tazobactam or avibactam [11]. While tigecycline shows excellent in vitro activity against CRAB, enthusiasm surrounding this agent has been tempered due to pharmacokinetic limitations of the drug, the development of resistance while on therapy, and its inability to demonstrate non-inferiority in the treatment of hospital-acquired and ventilator-associated pneumonia [14-17]. Due to the continued emergence and dissemination of resistant $A$. baumannii and the limited number of therapeutic options, other agents such as minocycline have been investigated for their utility in the treatment of these pathogens.

Minocycline is a second-generation tetracycline that was first introduced in the 1960s. While the oral formulation remained available, the intravenous formulation was taken off the US market in 2005 due to decreased use. It was re-introduced in 2009 and has become an important option for the treatment of multidrug-resistant organisms, in particular CRAB. Following reintroduction of minocycline to the market for the treatment of serious infections, including an FDA-approved indication for infections caused by Acinetobacter, there has been renewed interest in its use. Further investigation of its use for the treatment of MDR Acinetobacter infections, including those caused by carbapenem-resistant and XDR strains, has occurred due to its in vitro activity against $A$. baumannii, more favorable pharmacokinetics compared to tigecycline, and its safety profile. This review will focus on the tetracycline mechanisms of action and resistance, the in vitro activity of minocycline in the presence and absence of these resistance mechanisms, the pharmacokinetics and pharmacodynamics of minocycline, and finally the clinical experience with minocycline for the treatment of invasive infections due to $A$. baumannii.

\section{Compliance with Ethics Guidelines}

This article is based on previously conducted studies and does not involve any new studies of human or animal subjects performed by any of the authors.

\section{MECHANISMS OF ACTION AND RESISTANCE}

Tetracyclines are a broad-spectrum class of antimicrobials, including tetracycline, doxycycline, minocycline and tigecycline, which enter Gram-negative organisms through outer membrane protein channels and cause conformational changes to the RNA by binding with the $30 \mathrm{~S}$ ribosomal unit. Tetracyclines bind where the codon of the mRNA is recognized by the anticodon of the tRNA [18]. This binding blocks the entry of aminoacyl transfer RNA into the site A of the ribosome, which prevents elongation of the peptide chain [12]. It has been observed that alterations to the hydrophilic surface of the tetracycline molecule interferes with the antimicrobial activity of the drug, while alterations in the hydrophobic surface of the drug are less likely to interfere [18]. Doxycycline and minocycline are the more lipophilic counterparts of tetracycline, which allows for increased tissue penetration, increased antibacterial activity, longer half-lives and broader spectrums of activity against many bacterial species, including Acinetobacter species [12, 19-21].

Resistance to tetracyclines is conferred through a variety of mechanisms, including efflux pumps, ribosomal protection proteins, chemical molecule modification and target site modifications. Among Gram-negative organisms, the main mechanism leading to tetracycline resistance is through efflux pumps. The differences in gene-encoding efflux proteins account for the differences in resistance phenotypes to the different agents within the tetracycline class. There are over 20 efflux pump encoding genes that have been detected in Gram-negative organisms, with the most common being TetA. These efflux pumps lead to a decrease in tetracycline intracellular concentration by exchanging a proton for the tetracycline cation complex [12, 18]. In vitro efflux pumps seen in $A$. baumannii are effective in 
transporting out tetracycline and doxycycline, but with the exception of the TetB pump, not minocycline. Recent work by Lomoskaya and colleagues confirmed that the absence of TetB in $A$. baumannii has a negative predictive value of $100 \%$ for minocycline resistance $(93 / 93$ TetB negative strains were minocycline susceptible), whereas the presence of TetB has a $93 \%$ positive predictive value for minocycline resistance, with only 11/165 (6.7\%) isolates being susceptible to minocycline when TetB was present [22]. Thus, the gene encoding for TetB represents a potential target for rapid diagnostic gene testing to quickly determine minocycline susceptibility.

Tigecycline was synthetically designed to overcome these efflux pumps and has activity if either TetA or TetB is present. Unfortunately, although tigecycline was developed to overcome many of the efflux pump resistance mechanisms commonly seen with tetracyclines, rapid development of different resistance mechanisms have been observed. Current literature suggests that the development of such resistance has been associated with the Tet $X$ gene and, perhaps most importantly for $A$. baumannii, the overexpression of various efflux pumps (AdeABC, AdeIJK, AdeFGH, AbeM, AdeDE) [23, 24].

Resistance-nodulation-division (RND)-type efflux pumps, such as AdeABC, have been closely associated with the development of tigecycline resistance. These pumps have been shown to be more efficient than other types of efflux pumps in that they export drugs into the extracellular, rather than periplasmic, space. Up-regulation of these AdeABC pumps have been associated with higher MICs and increased resistance to tigecycline in vitro. Data from Hornsey and colleagues reported overexpression of the AdeABC efflux pump in two cases of tigecycline resistance, which emerged after tigecycline therapy, as well as a laboratory mutant with an elevated MIC. In this study, susceptibility was restored in a resistant isolate by interrupting the AdeB, which further supports a role of AdeABC in tigecycline resistance [25].

Interestingly, the RND pumps previously mentioned do not appear to impact minocycline susceptibility, and therefore tigecycline-resistant $A$. baumannii can remain susceptible to minocycline. Importantly, these RND pumps can be selected with clinically relevant concentrations of tigecycline (but not minocycline) and serve as a mechanism for the well-described development of tigecycline resistance while on therapy [26].

\section{In Vitro Activity}

It has been shown that minocycline has increased activity against MDR Acinetobacter compared to other early generation tetracyclines because of its ability to overcome many of the tetracycline resistance mechanisms (most notably TetA). A. baumannii containing oxacilinases or metalo-beta-lactamase genes has been reported to retain a susceptibility profile to minocycline similar to organisms without these genes [27]. In one in vitro study, using CLSI breakpoints, minocycline displayed a $30 \%$ improved susceptibility compared to doxycycline and nearly 60\% improved susceptibility compared to tetracycline against A. baumannii [12]. Thus, minocycline susceptibility should be determined by testing directly and not be determined by a surrogate class approach (i.e. tigecycline and doxycycline resistance does not necessarily equal minocycline resistance).

In vitro, minocycline has frequently been shown to be one of the only available treatment options for difficult to treat multi drug-resistant Acinetobacter infections, and as described above is often the second most active agent behind colistin. The antimicrobial activity of minocycline against $A$. baumannii was recently evaluated in two large surveillance studies from different parts of the world: the SENTRY antimicrobial surveillance program (2007-2011) [12] and the Tigecycline Evaluation and Surveillance Trial (T.E.S.T, 2005-2011) [28]. In the SENTRY program, 79.1\% of $A$. baumannii were susceptible to minocycline (second only to colistin, which had a $98.8 \%$ susceptibility rate), while in the T.E.S.T., A. baumannii had a susceptibility rate of $84.1 \%$, ranging between $68.5 \%$ in East South Central US and 97.4\% in New England. Similarly, multidrug-resistant $A$. baumannii (defined as resistance to 3 or more classes of antibiotics among beta-lactams, 
aminoglycosides, carbapenems or fluoroquinolones) were susceptible to minocycline in $72.1 \%$ of cases, (ranging between $54 \%$ in the East South Central US, and 92.3\% in New England).

Furthermore, a study by Castanheira and colleagues assessed A. baumannii isolates collected between 2007 and 2011 and showed that the only two antimicrobials with greater than $50 \%$ susceptibility rates were minocycline and colistin. Reports of minocycline retaining its activity in patient isolates that have developed colistin resistance during colistin therapy also exist [29]. Importantly, minocycline susceptibility remains high and is not impacted by carbapenem resistance. Colton and colleagues reported that isolates retained $\mathrm{MIC}_{50}$ values of $\leq 4.0 \mu \mathrm{g} / \mathrm{mL}$ for minocycline against Acinetobacter species, including carbapenem-resistant isolates [30]. A study performed in Thailand in CRAB confirmed these results with findings of $\mathrm{MIC}_{50}$ values of $4 \mathrm{mg} / \mathrm{L}$ and $\mathrm{MIC}_{90}$ values of $8 \mathrm{mg} / \mathrm{L}$ for minocycline, with $81.4 \%$ of isolates being susceptible [31].

Additionally, it is important to note that minocycline has also displayed high levels of synergistic activity with polymyxins in minocycline-resistant isolates, where $0.5 \mathrm{mg} / \mathrm{L}$ of polymyxin B restored in vitro susceptibility to minocycline in 167 tested isolates, $88 \%$ of which were resistant to minocycline monotherapy [32]. This is an important and encouraging finding given the propensity to use combination regimens in the treatment of CRAB.

\section{PHARMACOKINETICS AND PHARMACODYNAMICS}

In comparison to other more recently evaluated antimicrobials, there exist minimal data relating to the pharmacokinetics of minocycline. Studies which have assessed the pharmacokinetics of minocycline have found that serum concentrations are similar to other tetracyclines. Receipt of a 200-mg one-time intravenous dose has demonstrated peak serum concentrations ranging from 3 to $8.75 \mu \mathrm{g} / \mathrm{mL}$ and trough concentrations from 0.6 to $1.9 \mu \mathrm{g} /$ $\mathrm{mL}[21,33,34]$. After multiple oral doses of
$100 \mathrm{mg}$ every $12 \mathrm{~h}$, serum concentrations have ranged from approximately 0.7 to $3.9 \mu \mathrm{g} / \mathrm{mL}$ $[21,34,35]$. Similarly, data reported in the package insert for intravenous minocycline state peak and trough concentrations of $2.52-6.63$ and $0.82-2.64 \mu \mathrm{g} / \mathrm{mL}$, respectively [36]. The protein binding of minocycline is similar to that of tetracycline, with approximately $76 \%$ of the drug being protein-bound $[30,33]$. The half-life of minocycline following a single dose of either $200 \mathrm{mg}$ or $100 \mathrm{mg}$ is approximately $12-24 \mathrm{~h}$, which remains clinically unchanged in patients with renal dysfunction $[21,33,34,37]$. Minocycline is primarily metabolized by the liver and excreted through the hepatobiliary system, with only a small amount of drug eliminated through the renal route $(5-12 \%)$ unchanged in the urine $[33,37]$. For these reasons, it has been hypothesized that minocycline elimination is independent of renal function and hepatic function.

In a study looking at single intravenous doses and repeated oral doses of minocycline, it was reported that there was no evidence of reduced drug clearance in patients with reduced renal function, but a possible increase in tissue distribution [33]. This particular study evaluated 13 male patients with varying degrees of renal dysfunction, although none were on dialysis. The authors found that the decline in individual serum levels of minocycline activity after intravenous infusion was biphasic and therefore the pharmacokinetics were consistent with a two-compartment model. Both the concentration of antibiotic in urine and the cumulative renal excretion were directly related to kidney function, while the overall clearance of antibiotic appeared to be independent of renal function. As minocycline does not seem to accumulate in renal failure, it is believed that minocycline can be safely administered to patients with renal insufficiency without requiring any dose adjustments [33].

While minocycline has not been shown to accumulate in renal failure, there exists the notion that minocycline use in patients with renal insufficiency may enhance uremia seen in these patients due to the anti-anabolic processes associated with the drug on mammalian cells [33]. Although publications have suggested an 
increase in urea clearance and rises in plasma urea in patients with renal insufficiency receiving minocycline, it is unclear in these analyses whether or not these were related to antianabolic actions of the drug or simply a worsening of the patients' renal failure [27]. Authors have suggested that there is a critical level of free tetracycline that may be associated with increased urea production and that this level "is seldom reached at doses of $200 \mathrm{mg}$ /day of minocycline" [34]. These findings and statements serve as the basis for the package insert for minocycline carrying a warning to not exceed a total daily dosage of $200 \mathrm{mg}$ in $24 \mathrm{~h}$ in patients with renal impairment $(\mathrm{CrCl}<80 \mathrm{~mL}$ / min) [36]. However, assuming that a minocycline dose of $200 \mathrm{mg}$ BID is needed, based on pharmacokinetic and pharmacodynamic properties (described below), we would recommend against dose adjustment downward in these patients due to concerns of underexposure (since the half-life and presumably clearance of the drug would not be expected to change). Rather, we would suggest that clinicians be aware of potential antianabolic actions and closely monitor blood urea nitrogen levels and for signs/symptoms of uremia, given the serious nature of infections due to $A$. baumannii and the need to meet PK/PD targets.

Minocycline achieves high tissue penetration and displays excellent oral bioavailability allowing for expanded clinical uses by giving providers the ability to easily switch between the intravenous and oral formulations. Minocycline is the most lipophilic of all the tetracyclines because it has a greater partial coefficient at a neutral $\mathrm{pH}$, and therefore is believed to be the most potent, with a longer half-life, better oral absorption and enhanced tissue penetration compared to other tetracyclines. Previous literature has shown that minocycline has approximately a 20 -fold higher affinity to the ribosome than tetracycline and in vitro can inhibit translation 2-7 times more efficiently than tetracycline $[18,38,39]$.

The minocycline-free area under the concentration time curve to MIC ratio (fAUC/MIC) is the pharmacokinetic/pharmacodynamic parameter best associated with effect. Recent work by Tarazi and colleagues have identified the free AUC/MIC target to be associated with bacteriostasis and 1 log bactericidal activity. In this analysis, the authors explored exposure response with 6 different $A$. baumannii isolates (minocycline MIC's ranging from 0.03 to $4 \mathrm{mcg} / \mathrm{mL}$ ) in a rat pneumonia model. In this model, mean fAUC/MIC values associated with stasis and $1 \log$ kill were $12.2(10.6-16.1)$ and 18.0 (13.1-24.2) $\mathrm{mg} \mathrm{h} / \mathrm{L}$, respectively [40].

In order to interpret these fAUC/MIC values, it is essential to evaluate them in the perspective of achievable free AUCs obtained with approved dosing strategies. As has been previously stated in this section, scant pharmacokinetic data of minocycline are available, and no studies overtly evaluate AUC exposures. However, the analysis by Welling and colleagues published 40 years ago does report on clearance rates in patients with normal renal function and various degrees of renal insufficiency [26]. Interestingly, in this analysis, they found the clearance rate was the lowest in patients with normal renal function $(1.18 \mathrm{~L} / \mathrm{h})$, and slightly higher in patients with renal insufficiency $(1.84-2.01 \mathrm{~L} / \mathrm{h})$. Using the basic pharmacokinetic equation Dose $=$ AUC/Clearance, and the known protein binding of $\sim 76 \%$, one can estimate free AUC exposures and ultimately AUC/ MIC ratios with various minocycline dosing regimens for different organism MICs. For patients receiving 200-400 mg daily of minocycline, total area under the concentration time curve exposures would be expected to range from 100 to $340 \mathrm{mg} \mathrm{h} / \mathrm{L}$, resulting in a free AUC of $24-82 \mathrm{mg} \mathrm{h} / \mathrm{L}$. Thus, even in the worst case scenario, where the highest clearance rate $(2.01 \mathrm{~L} / \mathrm{h})$ and highest MIC in the susceptible range $(4 \mathrm{mcg} / \mathrm{mL})$ is utilized, a dose of $200 \mathrm{mg}$ BID (total AUC $=199 \mathrm{mg} \mathrm{h} / \mathrm{L}$, free AUC $48 \mathrm{mg} \mathrm{h} / \mathrm{L}$ ) would be expected to achieve bacteriostasis (fAUC/MIC $=12$ ), and in most scenarios bactericidal activity would be expected.

Importantly, however, these data are limited in their interpretability given the large variance in clearance rates reported, the use of $200-\mathrm{mg}$ daily doses (as opposed to 400-mg daily doses), and the small study sample sizes. Additionally, even though minocycline is considered highly bioavailable with $\sim 90 \%$ being orally absorbed, it is unknown if there were confounding factors 
impacting absorption in this study, and it is possible that higher doses and intravenous administration could lead to increased exposures further leading to enhanced bactericidal activity. Although modern pharmacokinetic data are urgently needed for minocycline administered in both its oral and intravenous formulations, data currently available are encouraging. Importantly, additional PK studies of IV minocycline in normal subjects, critically-ill patients and infected patients, and in patients with chronic renal impairment are planned (Mike Dudley, personal communication).

\section{CLINICAL EXPERIENCE OF MINOCYCLINE FOR MDR GRAM-NEGATIVE BACILLI}

\section{Overview of Clinical Data}

Currently, there have been no randomized, controlled trials that evaluate the efficacy of minocycline in treatment of MDR Gram-negative bacterial infections; however, clinical data provide evidence of its potential role in the treatment of these difficult to treat pathogens. Between the years 1998 and 2015, seven retrospective studies [16, 41-47] evaluated the use of oral or intravenous minocycline alone or in combination with other antimicrobials for the treatment of MDR A. baumannii. One factor complicating interpretation of these studies is the variety of different definitions used by different investigators. MDR A. baumannii was defined as resistance to 3 or more antimicrobial classes in one study [42] and resistance to all frequently tested beta-lactams in another study [44]. CRAB was defined as carbapenem-resistant $[16,45]$ and pan-drug-resistant $A$. baumannii was defined as $A$. baumannii resistant to all antibiotics excluding polymyxins [46].

The seven studies included 126 patients between 17 and 85 years old (Table 1), who had 141 identifiable isolates that included the following sources: respiratory tract $(91,74.6 \%)$, blood $(22,18 \%)$, bone $(11,9 \%)$, skin and soft tissue (including surgical site infections) (12,
$9.8 \%)$, urine $(2,1.6 \%)$, and others $(3,2.4 \%)$. Of the patients treated, 94 patients received minocycline combined with another antimicrobial agent, 12 patients were treated with minocycline monotherapy, and 11 patients received monotherapy with either minocycline or doxycycline, with the exact agent not specified [40]. A majority of the patients (72.5\%) received minocycline intravenously. The most common dose used was $100 \mathrm{mg}$ twice daily, with or without a loading dose of $200 \mathrm{mg}$ (overall, 61 subjects received a loading dose). One study included patients treated with IV minocycline $200 \mathrm{mg}$ twice daily [16], and in another study patients treated with oral

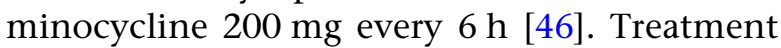
was administered for a duration that ranged between 2 days to 7 weeks, according to the source of infection [44]. Median durations of therapy from each study is presented in Table 1. Overall, the clinical success rate of monotherapy or combination treatment was $78.2 \%$. Microbiological cure (defined differently across studies) was reported in 4 studies and ranged from $50 \%$ and $89 \%$ (Table 1).

\section{Clinical Data for Monotherapy}

Twenty-three patients in five of the studies were treated with either minocycline or doxycycline as monotherapy for $A$. baumannii infection [42-46]. As previously stated, in one study there was no differentiation between treatment with minocycline and doxycycline, and therefore the data are presented together [40]. The age of the patients ranged between 15 and 85 years (Table 1). Of the 22 known sources of $A$. baumannii isolates, $16(73 \%)$ were from the respiratory tract, $4(18 \%)$ from soft tissues, and 2 (9\%) from the bone. There were no bloodstream infections in this group. The route of minocycline administration was reported in 12 patients and was intravenous in most cases $(92 \%)$. The most commonly used dose was $100 \mathrm{mg}$ twice daily, although one study treated patients with $200 \mathrm{mg}$ BID. The number of subjects who received a loading dose could not be determined. The duration of treatment ranged between 2 days for an unknown source and 


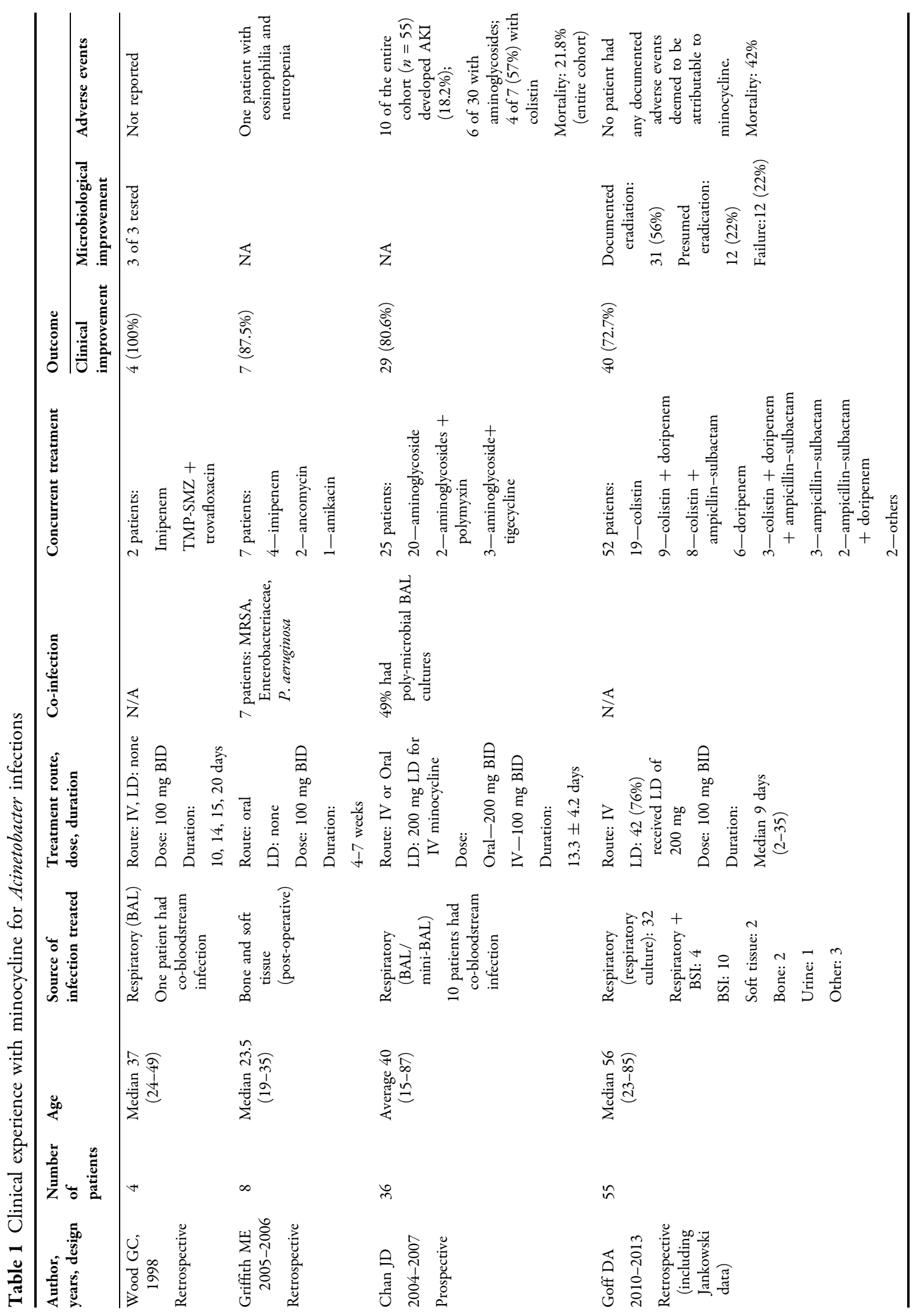




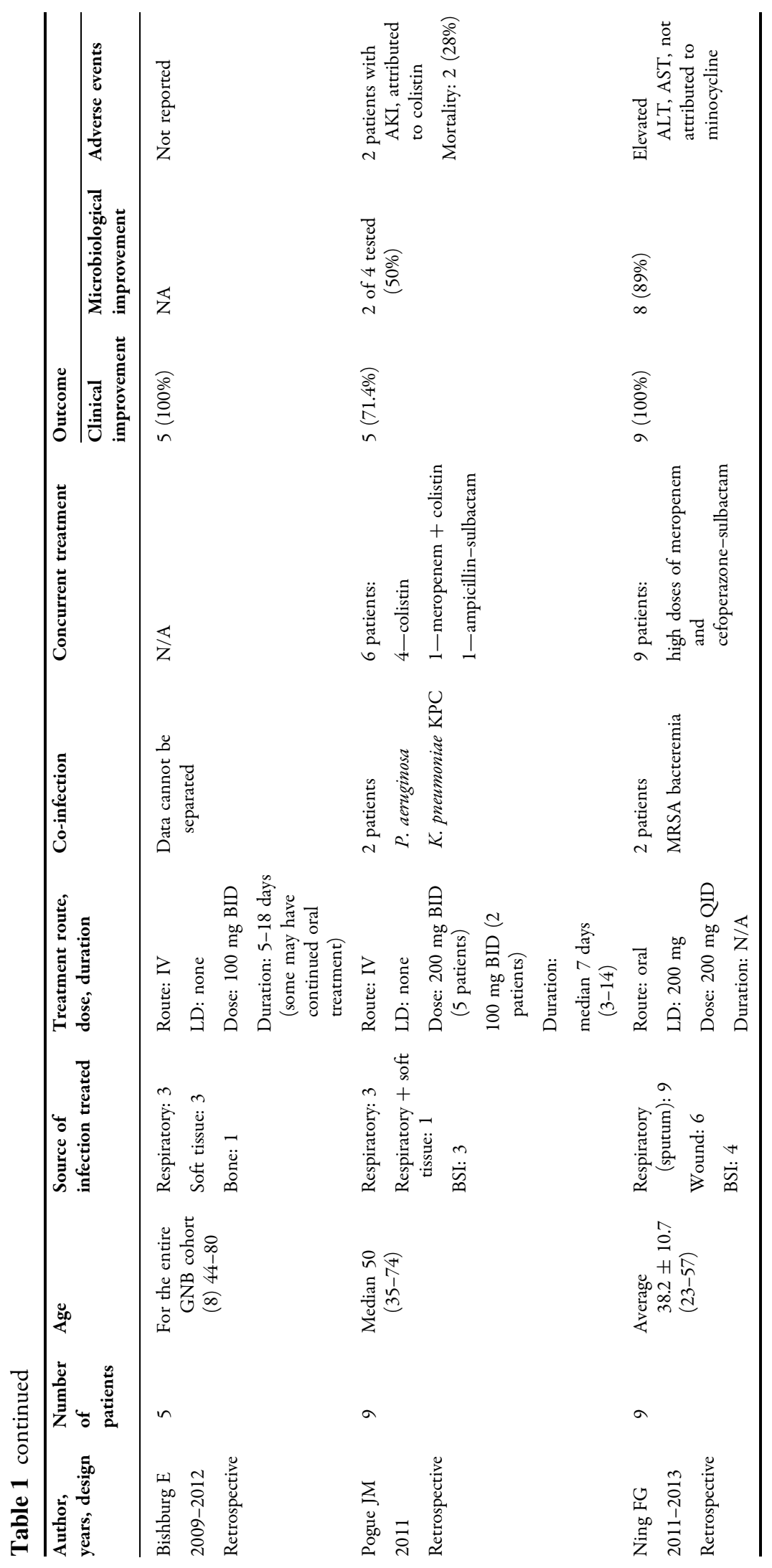


6 weeks in post-fracture infections. In one study, clinical success was achieved in $81.8 \%$ of subjects [40] and in 100\% of subjects in the four other studies. Microbiological cure was not available in this group.

\section{Adverse Events Data in All Clinical Studies}

Adverse events were reported in five studies $[16,42,44,45,47]$. In one study, a patient developed eosinophilia and neutropenia [44]. Two studies reported acute kidney injury (occurring in $28 \%$ and $18.2 \%$ of subjects) which was attributed to concomitant colistin or aminoglycoside treatment $[16,45]$. One study reported elevated liver enzymes which were attributed to host factors (severity of illness) and not to the minocycline [46]. Two other studies reported no adverse reactions or events attributed to minocycline [42].

\section{DISCUSSION}

Acinetobacter species are common nosocomial pathogens that have been implicated in serious infections associated with high morbidity and mortality rates. Historically, carbapenems were used to treat MDR Acinetobacter infections, but in recent years an increase in CRAB has decreased the therapeutic effectiveness of these agents. Limitations of available treatment options have left clinicians in need of additional therapeutic choices. During the last decade, the interest in minocycline has increased due to its in vitro activity against $A$. baumannii (including MDR and XDR strains), as well as its pharmacokinetic and pharmacodynamic properties (e.g., its ability to achieve good serum and tissue levels as well as to display bactericidal activity). The ability of minocycline to overcome many resistance mechanisms that decrease the activity of other tetracyclines, most notably TetA and RND pumps, has allowed for its more widespread use against MDR Acinetobacter. Its favorable safety profile, lack of needed dose adjustments for renal and hepatic failure, and the easy conversion between IV to PO formulations have enhanced its clinical appeal. This is particularly true for difficult to manage patients with resistant Acinetobacter infections where available data support therapeutic success in these challenging clinical scenarios. Given the paucity of antimicrobials currently available to treat MDR and XDR A. baumannii, further clinical and laboratory evaluation of minocycline as an effective treatment alternative for infections caused by resistant pathogens is warranted. Additionally, further studies to assess if minocycline monotherapy is sufficient or whether it should be utilized as part of a combination therapeutic regimen are needed.

\section{ACKNOWLEDGEMENTS}

No funding or sponsorship was received for this study or publication of this article. All named authors meet the International Committee of Medical Journal Editors (ICMJE) criteria for authorship for this manuscript, take responsibility for the integrity of the work as a whole, and have given final approval for the version to be published.

Disclosures. Jason M. Pogue has served as a consultant for The Medicines Company. Keith S. Kaye has served as a consultant for The Medicines Company. Jennifer N. Lashinsky and Oryan Henig have nothing to disclose.

Compliance with Ethics Guidelines. This article is based on previously conducted studies and does not involve any new studies of human or animal subjects performed by any of the authors.

Data Availability. Data sharing is not applicable to this article as no datasets were generated or analyzed during the current study.

Open Access. This article is distributed under the terms of the Creative Commons Attribution-NonCommercial 4.0 International License (http://creativecommons.org/licenses/ by-nc/4.0/), which permits any noncommercial use, distribution, and reproduction in any medium, provided you give appropriate credit to the original author(s) and the source, provide 
a link to the Creative Commons license, and indicate if changes were made.

\section{REFERENCES}

1. Falagas ME, Koletsi PK, Bliziotis IA. The diversity of definitions of multidrug-resistant (MDR) and pandrug-resistant (PDR) Acinetobacter baumannii and Pseudomonas aeruginosa. J Med Microbiol. 2006;55(Pt 12):1619-29.

2. Falagas ME, Bliziotis IA, Siempos II. Attributable mortality of Acinetobacter baumannii infections in critically ill patients: a systematic review of matched cohort and case-control studies. Crit Care. 2006;10(2):R48.

3. Villegas MV, Hartstein AI. Acinetobacter outbreaks, 1977-2000. Infect Control Hosp Epidemiol. 2003;24(4):284-95.

4. Dijkshoorn L, Nemec A, Seifert H. An increasing threat in hospitals: multidrug-resistant Acinetobacter baumannii. Nat Rev Microbiol. 2007;5(12):939-51.

5. Centers for Disease Control and Prevention. Tracking CRE Infections. 2013. http://www.cdc.gov/hai/ organisms/cre/TrackingCRE.html. Accessed Oct 2016.

6. Kim UJ, Kim HK, An JH, Cho SK, Park KH, Jang HC. Update on the epidemiology, treatment, and outcomes of carbapenem-resistant acinetobacter infections. Chonnam Med J. 2014;50(2):37-44.

7. Reddy T, Chopra T, Marchaim D, Pogue JM, Alangaden $\mathrm{G}$, Salimnia $\mathrm{H}$, et al. Trends in antimicrobial resistance of Acinetobacter baumannii isolates from a metropolitan Detroit health system. Antimicrob Agents Chemother. 2010;54(5):2235-8.

8. Cheah SE, Wang J, Nguyen VT, Turnidge JD, Li J, Nation RL. New pharmacokinetic/pharmacodynamic studies of systemically administered colistin against Pseudomonas aeruginosa and Acinetobacter baumannii in mouse thigh and lung infection models: smaller response in lung infection. J Antimicrob Chemother. 2015;70(12):3291-7.

9. Queenan AM, Pillar CM, Deane J, Sahm DF, Lynch AS, Flamm RK, et al. Multidrug resistance among Acinetobacter spp. in the USA and activity profile of key agents: results from CAPITAL Surveillance 2010. Diagn Microbiol Infect Dis. 2012;73(3):267-70.

10. Oikonomou O, Sarrou S, Papagiannitsis CC, Georgiadou S, Mantzarlis K, Zakynthinos E, et al. Rapid dissemination of colistin and carbapenem resistant Acinetobacter baumannii in Central Greece: mechanisms of resistance, molecular identification and epidemiological data. BMC Infect Dis. 2015;15:559.

11. Zilberberg MD, Kollef MH, Shorr AF. Secular trends in Acinetobacter baumannii resistance in respiratory and blood stream specimens in the United States, 2003 to 2012: a survey study. J Hosp Med. 2016;11(1):21-6.

12. Castanheira M, Mendes RE, Jones RN. Update on Acinetobacter species: mechanisms of antimicrobial resistance and contemporary in vitro activity of minocycline and other treatment options. Clin Infect Dis. 2014;59(Suppl 6):S367-73.

13. Vidal L, Gafter-Gvili A, Borok S, Fraser A, Leibovici L, Paul M. Efficacy and safety of aminoglycoside monotherapy: systematic review and meta-analysis of randomized controlled trials. J Antimicrob Chemother. 2007;60(2):247-57.

14. Freire AT, Melnyk V, Kim MJ, Datsenko O, Dzyublik $\mathrm{O}$, Glumcher $\mathrm{F}$, et al. Comparison of tigecycline with imipenem/cilastatin for the treatment of hospital-acquired pneumonia. Diagn Microbiol Infect Dis. 2010;68(2):140-51.

15. Pogue JM, Mann T, Barber KE, Kaye KS. Carbapenem-resistant Acinetobacter baumannii: epidemiology, surveillance and management. Expert Rev Anti Infect Ther. 2013;11(4):383-93.

16. Pogue JM, Neelakanta A, Mynatt RP, Sharma S, Lephart P, Kaye KS. Carbapenem-resistance in gram-negative bacilli and intravenous minocycline: an antimicrobial stewardship approach at the Detroit Medical Center. Clin Infect Dis. 2014;59(Suppl 6):S388-93.

17. Bhavnani SM, Rubino CM, Hammel JP, Forrest A, Dartois N, Cooper CA, et al. Pharmacological and patient-specific response determinants in patients with hospital-acquired pneumonia treated with tigecycline. Antimicrob Agents Chemother. 2012;56(2):1065-72.

18. Nguyen F, Starosta AL, Arenz S, Sohmen D, Donhofer A, Wilson DN. Tetracycline antibiotics and resistance mechanisms. Biol Chem. 2014;395(5):559-75.

19. Dimitriadis P, Protonotariou E, Varlamis S, Poulou A, Vasilaki O, Metallidis S, et al. Comparative evaluation of minocycline susceptibility testing methods in carbapenem-resistant Acinetobacter baumannii. Int $\mathrm{J}$ Antimicrob Agents. 2016;48(3):321-3.

20. Falagas ME, Vardakas KZ, Kapaskelis A, Triarides NA, Roussos NS. Tetracyclines for 
multidrug-resistant Acinetobacter baumannii infections. Int $\mathrm{J}$ Antimicrob Agents. 2015;45(5): 455-60.

21. Macdonald H, Kelly RG, Allen ES, Noble JF, Kanegis LA. Pharmacokinetic studies on minocycline in man. Clin Pharmacol Ther. 1973;14(5):852-61.

22. Lomovskaya O SD, Rubio-Aparicio D, Nelson K, Dudley M. TetB testing and its absence identifies minocycline (MINO) susceptible isolates of Acinetobacter baumannii (ACB). In: Presented at: IDWeek; October 26-30, 2016; New Orleans, LA.

23. Montana S, Vilacoba E, Traglia GM, Almuzara M, Pennini M, Fernandez A, et al. Genetic variability of AdeRS two-component system associated with tigecycline resistance in XDR-Acinetobacter baumannii isolates. Curr Microbiol. 2015;71(1):76-82.

24. Pournaras S, Koumaki V, Gennimata V, Kouskouni E, Tsakris A. In vitro activity of tigecycline against Acinetobacter baumannii: global epidemiology and resistance mechanisms. Adv Exp Med Biol. 2016;897:1-14.

25. Hornsey M, Ellington MJ, Doumith M, Thomas CP, Gordon NC, Wareham DW, et al. AdeABC-mediated efflux and tigecycline MICs for epidemic clones of Acinetobacter baumannii. J Antimicrob Chemother. 2010;65(8):1589-93.

26. Sun D R-AD, Tsivkovski R, King P, Dudley M, Lomovskaya O. Tigecycline (TIG) but NOT Minocycline (MINO) Readily selects for clinically relevant efflux-mediated resistance (R) in acinetobacter (ACB). In: Presented at: 53rd Interscience Conference on Antimicrobial Agents and Chemotherapy; September 10-13, 2013; Denver, CO.

27. Rizek C, Ferraz JR, van der Heijden IM, Giudice M, Mostachio AK, Paez J, et al. In vitro activity of potential old and new drugs against multidrug-resistant gram-negatives. J Infect Chemother. 2015;21(2):114-7.

28. Denys GA, Callister SM, Dowzicky MJ. Antimicrobial susceptibility among gram-negative isolates collected in the USA between 2005 and 2011 as part of the Tigecycline Evaluation and Surveillance Trial (T.E.S.T.). Ann Clin Microbiol Antimicrob. 2013;12:24.

29. Qureshi ZA, Hittle LE, O'Hara JA, Rivera JI, Syed A, Shields RK, et al. Colistin-resistant Acinetobacter baumannii: beyond carbapenem resistance. Clin Infect Dis. 2015;60(9):1295-303.

30. Colton B, McConeghy KW, Schreckenberger PC, Danziger LH. I.V. minocycline revisited for infections caused by multidrug-resistant organisms. Am J Health Syst Pharm. 2016;73(5):279-85.
31. Thamlikitkul V, Tiengrim S, Seenama C. Comparative in vitro activity of minocycline and selected antibiotics against carbapenem-resistant Acinetobacter baumannii from Thailand. Int J Antimicrob Agents. 2016;47(1):101-2.

32. Lomovskaya O NK, Rubio-Aparicio D, Sun D, Giffith DC, Dudley MN. Minocycline acitivity is enhanced by polymyxin $\mathrm{B}$ in tetB-containing isolates of Acinetobacter baumannii. In: Presented at: IDWeek; October 26-30, 2016; New Orleans, LA.

33. Welling PG, Shaw WR, Uman SJ, Tse FL, Craig WA. Pharmacokinetics of minocycline in renal failure. Antimicrob Agents Chemother. 1975;8(5):532-7.

34. Carney S, Butcher RA, Dawborn JK, Pattison G. Minocycline excretion and distribution in relation to renal function in man. Clin Exp Pharmacol Physiol. 1974;1(4):299-308.

35. Maesen FP, Davies BI, van den Bergh JJ. Doxycycline and minocycline in the treatment of respiratory infections: a double-blind comparative clinical, microbiological and pharmacokinetic study. J Antimicrob Chemother. 1989;23(1):123-9.

36. Minocin $^{\circledR}$ (Minocycline), USP [package insert]. Monza (Milan), Italy: Triax Pharmaceuticals; 2010.

37. Sklenar I, Spring P, Dettli L. One-dose and multiple-dose kinetics of minocycline in patients with renal disease. Agents Actions. 1977;7(3):369-77.

38. Bergeron J, Ammirati M, Danley D, James L, Norcia M, Retsema J, et al. Glycylcyclines bind to the high-affinity tetracycline ribosomal binding site and evade Tet(M)- and Tet(O)-mediated ribosomal protection. Antimicrob Agents Chemother. 1996;40(9):2226-8.

39. Olson MW, Ruzin A, Feyfant E, Rush TS 3rd, O'Connell J, Bradford PA. Functional, biophysical, and structural bases for antibacterial activity of tigecycline. Antimicrob Agents Chemother. 2006;50(6):2156-66.

40. Tarazi ZSM, Dudley MN, Griffith DC. Pharmacodynamics of minocycline against Acinetobacter baumannii in a rat pneumonia model. In: Presented at: 55th Interscience Conference on Antimicrobial Agents and Chemotherapy; September 18-21, 2015; San Diego, CA.

41. Jankowski CA, Balada-Llasat J-M, Raczkowski M, Pancholi P, Goff DA. A stewardship approach to combating multidrug-resistant Acinetobacter baumannii infections with minocycline. Infect Dis Clin Pract 2012;20:4.

42. Goff DA, Bauer KA, Mangino JE. Bad bugs need old drugs: a stewardship program's evaluation of 
minocycline for multidrug-resistant Acinetobacter baumannii infections. Clin Infect Dis. 2014;59(Suppl 6):S381-7.

43. Wood GC, Hanes SD, Boucher BA, Croce MA, Fabian TC. Tetracyclines for treating multidrug-resistant Acinetobacter baumannii ventilator-associated pneumonia. Intensive Care Med. 2003;29(11):2072-6.

44. Griffith ME, Yun HC, Horvath LL, Murray CK. Minocycline therapy for traumatic wound infections caused by the multidrug-resistant Acinetobacter baumannii-Acinetobacter calcoaceticus Complex. Infect Dis Clin Pract. 2008;16:4.

45. Chan JD, Graves JA, Dellit TH. Antimicrobial treatment and clinical outcomes of carbapenem-resistant Acinetobacter baumannii ventilator-associated pneumonia. J Intensive Care Med. 2010;25(6):343-8.

46. Bishburg E, Bishburg K. Minocycline-an old drug for a new century: emphasis on methicillin-resistant Staphylococcus aureus (MRSA) and Acinetobacter baumannii. Int $\mathrm{J}$ Antimicrob Agents. 2009;34(5):395-401.

47. Ning F, Shen Y, Chen X, Zhao X, Wang C, Rong Y, et al. A combination regimen of meropenem, cefoperazone-sulbactam and minocycline for extensive burns with pan-drug resistant Acinetobacter baumannii infection. Chin Med J. 2014;127(6):1177-9. 